\title{
Monográfico: Formación del profesorado de Música: de dónde venimos y hacia
} dónde vamos

Monographic: Music teacher training: where we come from and where we are going

$$
\begin{array}{r}
\begin{array}{r}
\text { Rosa M. Serrano } \\
\text { rmserran @ unizar.es }
\end{array} \\
\text { Departamento de Expresión Musical, Plástica y Corporal } \\
\text { Universidad de Zaragoza } \\
\text { Zaragoza, España }
\end{array}
$$

doi: 10.7203/LEEME.46.18977 Plástica y Corporal, Universidad de Zaragoza, C/ Pedro Cerbuna, 12, C.P. 50009 Zaragoza. España.

La formación del profesorado en general y de educación musical en particular es un tema de investigación recurrente a nivel mundial (Goodwin, 2010), ya que la calidad docente determina la calidad del sistema educativo (Darling-Hammond et al., 2017). En los últimos años, aspectos como la creación del Espacio Europeo de Educación Superior han conllevado cambios en los planes de estudio de la formación del profesorado, tanto en el ámbito nacional como internacional. En este sentido, se hace necesario analizar, desde una visión crítica, cómo han influido estas transformaciones en la formación del futuro profesorado de música y su adecuación al contexto actual. Para ello, en este monográfico se recogen ocho estudios que buscan dar respuesta a esta temática desde diferentes perspectivas.

Desde un primer enfoque, se presentan tres estudios centrados en el análisis de planes de estudio universitarios en el ámbito español. El trabajo de Antonio Fernández-Jiménez y Francisco A. Valdivia analiza en detalle la secuenciación, distribución de asignaturas y temporalización de los planes de estudio de la Mención en Educación Musical del Grado de Maestro en Educación Primaria en las universidades españolas; examinando un total de 47 planes de estudio existentes en todo el territorio. Por su parte, Rosa M. Serrano, Felipe Javier Zamorano y Cristina González-Martín se centran en el análisis de los planes de estudio del Máster en Formación del Profesorado de Música en Educación Secundaria y Bachillerato en España en relación a las demandas de la escuela y el aula de música en la actual sociedad y economía del conocimiento; en este caso, su análisis comparado (general y específico musical, en el que definen tipologías y sus implicaciones en la formación docente) es de un total de 26 planes de estudio. Desde una visión transversal de la formación del profesorado en el Grado y en el Máster, Yurima Blanco y Alicia Peñalba examinan los planes de estudio de los futuros docentes de música de las universidades de Castilla y León, realizando un profundo análisis de los contenidos musicales, contenidos transversales y objetivos de la educación de los ocho planes de estudio existentes en la Comunidad.

En el ámbito internacional, la aportación de Felipe Javier Zamorano analiza la identidad activista en la formación del profesorado de música en Chile, concluyendo que el plan de

@Rosa M. Serrano. The content of this article is the sole responsibility of the authors. The Revista Electrónica de LEEME and Universitat de València are not liable for any legal actions that may arise involving the article's content. Revista Electrónica de LEEME - Lista Electrónica Europea de Música en la Educación-. http://ojs.uv.es/index/php/LEEME/index ISSN: 1575-9563. Editores: Universidad de Valencia y Jesús Tejada. Visibilidad de esta revista: SCOPUS, Emerging Sources Citation Index (Clarivate), EBSCO, CINDOC (CSIC), Citefactor, COPAC, Dialnet, DICE (CSIC), DOAJ, e-revistas (CSIC), EBSCO Premier, ERIH+, Gale Cengage Learning, INRECS, IRESIE, LATINDEX, MIAR, OCLC Worldcat, RESH, REDIB, RILM Core Journals, SUDOC, ULRICHS. Esta revista es de acceso libre mediante licencia Creative Commons 4.0 CC by. Política de archivado: etiqueta verde SHERPA-ROMEO. 


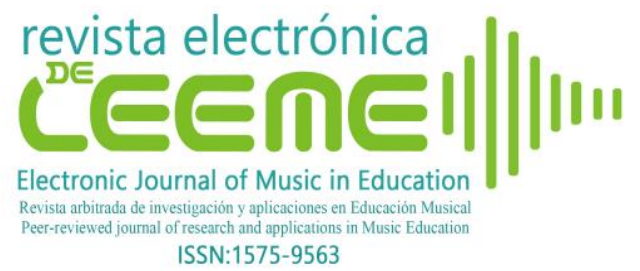

estudios permite que esta aflore gracias a la articulación de sus asignaturas y la promoción de estudiantes autónomos y proactivos, con una base pedagógica, crítica y reflexiva. A nivel europeo, Sara Domínguez y Margarita Pino estudian de manera comparada la formación inicial del profesorado de Primaria y Secundaria, a través de tres informes de investigación que permiten analizar las instituciones, duración, especialización, conocimientos previos y tipo de titulación de los estudios en los diferentes países. Finalmente, Martin Fautley analiza la formación del profesorado de música en Inglaterra, con especial énfasis en la preparación para enseñar el Currículo Nacional; realiza un profundo examen que propicia la reflexión a temáticas docentes de gran interés a nivel mundial, como la falta de reconocimiento o el tradicionalismo neo-conservador de la materia.

El tercer bloque queda vinculado al análisis de aspectos metodológicos y de praxis y su repercusión en la formación docente. Elena Berrón e Inés María Monreal abordan la formación inicial del futuro profesorado de Educación Primaria a través del Aprendizaje Basado en Proyectos desarrollado desde la educación musical. Conocer la opinión de los implicados, sus creencias sobre dicha metodología y su predisposición a utilizarla en su futura práctica docente es el objetivo del estudio. Por su parte, Alfredo Bautista y Basilio Fernández profundizan en el concepto y marco teórico de mala praxis en las enseñanzas instrumentales, resaltando las implicaciones que conlleva para la formación docente y la necesidad de su prevención.

Con esta visión amplia y diversificada se pretende ofrecer al lector nacional e internacional las bases para una reflexión profunda y crítica sobre distintas temáticas que afectan a la formación de docentes, que cuente con una base musical y pedagógica sólida, así como una disposición reflexiva, crítica, creativa y proactiva que dé respuesta a las demandas del siglo XXI. Todo ello teniendo en cuenta que la acción transformadora que se le exige a la educación debe comenzar en la formación inicial de quienes serán docentes (López-Pelaez, 2020).

\section{Referencias}

Darling-Hammond, L., Burns, D., Campbell, C., Goodwin, A.L., Hammerness, K., Low, E.L. y Zeichner, K. (2017). Empowered educators: How high-performing systems shape teaching quality around the world. New York: John Wiley \& Sons.

Goodwin, A.L. (2010). Globalization and the preparation of quality teachers: rethinking knowledge domains for teaching. Teaching Education, 21(1), 19-32. doi:10.1080/10476210903466901

López-Peláez, M.P. (2020). El profesorado de música ante la escuela del siglo XXI. Un reto para la formación en la Universidad. En A. Murillo et al. (Coords.), Escuelas creadoras. Escuelas del cambio (pp.141-154). Valencia: Edictoràlia. 